\title{
Chronic kidney disease in adults: assessment and management
}

\author{
Authors: Anna Forbes ${ }^{A}$ and Hugh Gallagher ${ }^{B}$
}

\begin{abstract}
Chronic kidney disease (CKD) is a common condition associated with significant amenable morbidity and mortality, primarily related to the substantially increased risk of cardiovascular disease (CVD) in this population. Early detection of people with CKD is important so that treatment can be initiated to prevent or delay kidney disease progression, reduce or prevent the development of complications, and reduce the risk of CVD. Classification of CKD by the estimated glomerular filtration rate and urine albumin to creatinine ratio identifies those at greatest risk of adverse outcomes. This concise guideline highlights the key recommendations of the National Institute for Health and Care Excellence guideline Chronic kidney disease in adults: assessment and management: Clinical guideline [CG182], published in July 2014. It focuses on recommendations most relevant to secondary care physicians.
\end{abstract}

KEYWORDS: Chronic kidney disease, NICE guidance, cardiovascular disease

DOI: $10.7861 /$ clinmed.cg.20.2

\section{Introduction}

Chronic kidney disease (CKD) is common, with an estimated prevalence in adults of $13 \%$ according to the Health Survey for England - 2009.1 The risk of CKD increases with age and the condition commonly coexists with hypertension, diabetes and cardiovascular disease (CVD). It is frequently unrecognised as there are often no specific symptoms. An important minority of people with CKD will develop end-stage kidney disease, and in this group late presentation for renal replacement therapy increases morbidity and mortality. However, the greatest significance of CKD is as an independent, powerful and potentially modifiable risk factor for CVD. ${ }^{2}$ CKD is also strongly associated with other important adverse outcomes, including acute kidney injury, frailty and mortality.

CKD is a growing healthcare challenge. In 2009-2010 CKD accounted for $1.3 \%$ of all NHS spending in that year; more than half this amount was spent on the $2 \%$ of people that progress to kidney failure. ${ }^{3}$ The estimated costs of excess strokes and myocardial

Authors: ${ }^{\text {A }}$ specialty registrar in nephrology, Frimley Health NHS Foundation Trust, Frimley, UK; ${ }^{\mathrm{B}}$ consultant nephrologist, Epsom and St Helier University Hospitals NHS Trust, Carshalton, UK infarctions (relative to an age and gender matched population without CKD) in the same year was between $£ 174-178$ million. ${ }^{3}$

Prompt diagnosis and management of CKD can prevent or delay progression and reduce the development of complications.

\section{Scope and purpose}

This concise guidance highlights key recommendation of the National Institute for Health and Care Excellence (NICE) guideline Chronic kidney disease in adults: assessment and management: Clinical guideline [CG182], published in July $2014 .{ }^{4}$

\section{Recommendations}

\section{Information and education}

People with CKD should be offered education and information tailored to the severity and cause of CKD, the associated complications and risk of progression.

Systems should be in place to inform people with CKD of their diagnosis, enable people with CKD to share in decision making about their care, and support self-management. This should include giving people access to their medical data and providing information about the importance of blood pressure control, smoking cessation, exercise, diet and medications.

\section{Diagnosis of CKD}

CKD is defined as an abnormality of kidney function and/or structure present for more than 3 months. This includes individuals with:

> markers of kidney damage including albuminuria, urine sediment abnormalities, electrolyte and other abnormalities due to tubular disorders, abnormalities detected by histology, structural abnormalities detected by imaging and a history of kidney transplantation

> glomerular filtration rate (GFR) $<60 \mathrm{~mL} / \mathrm{min} / 1.73 \mathrm{~m}^{2}$ on at least two occasions separated by at least 90 days (with or without kidney damage).

Clinical laboratories should use the CKD-EPI creatinine equation to estimate GFRcreatinine. In people with extremes of muscle mass, this should be interpreted with caution as reduced muscle mass will lead to overestimation and increased muscle mass to underestimation of the GFR.

Measurement of estimated GFRcystatinC (eGFRcystatinC) at initial diagnosis should be considered to confirm or rule out CKD in people with: 
> an eGFRcreatinine $45-59 \mathrm{~mL} / \mathrm{min} / 1.73 \mathrm{~m}^{2}$ sustained for at least 90 days and

$>$ no proteinuria (albumin to creatinine ratio (ACR) $<3 \mathrm{mg} / \mathrm{mmol}$ ) or no other marker of kidney disease.

CKD should not be diagnosed in people with:

> an eGFRcreatinine $45-59 \mathrm{~mL} / \mathrm{min} / 1.73 \mathrm{~m}^{2}$ and

$>$ an eGFRcystatinC $>60 \mathrm{~mL} / \mathrm{min} / 1.73 \mathrm{~m}^{2}$ and

$>$ no other marker of kidney disease.

Where a highly accurate measure of GFR is required such as during monitoring of chemotherapy or evaluation of renal function in potential living donors consider a reference standard measure (inulin, chromium complexed with ethylene diamine tetracetic acid, iohexol or 125I-iothalamate).

To identify proteinuria, urine ACR should be used in preference to protein to creatinine ratio (PCR) as it has a greater sensitivity than PCR for low levels of proteinuria. An ACR $3 \mathrm{mg} / \mathrm{mmol}$ or more is clinically important proteinuria. PCR can be used as an alternative to quantify and monitor higher levels of proteinuria (ACR of $70 \mathrm{mg} / \mathrm{mmol}$ or more).

\section{Who to test for CKD and who to refer for specialist} assessment

The major risk factors for CKD and criteria for specialist nephrology referral are summarised in Table 1.

Table 1. Who to test for chronic kidney disease and who to refer for specialist assessment

\section{Who to test for CKD (using GFR and ACR)}

Diabetes mellitus

Hypertension

Acute kidney injury

Cardiovascular disease

Structural renal tract disease

Multisystem diseases with potential kidney involvement eg SLE

Family history of end stage kidney disease or hereditary kidney disease

Opportunistic detection of haematuria

eGFR should also be monitored at least annually in people prescribed drugs known to be nephrotoxic, such as calcineurin inhibitors (for example, cyclosporin or tacrolimus), lithium and non-steroidal anti-inflammatory drugs. $\mathrm{ACR}=$ albumin to creatinine ratio; $\mathrm{CKD}=$ chronic kidney disease; GFR = glomerular filtration rate; SLE = systemic lupus erythematosus.

\section{Who to refer for specialist assessment}

GFR $<30 \mathrm{~mL} / \mathrm{min} / 1.73 \mathrm{~m}^{2}$ with or without diabetes

ACR $\geq 70 \mathrm{mg} / \mathrm{mmol}$ unless caused by diabetes and appropriately treated

ACR $\geq 30 \mathrm{mg} / \mathrm{mmol}$ together with haematuria

Sustained decrease in GFR $\geq 25 \%$ and a change in GFR category or sustained decrease in GFR $\geq 15 \mathrm{~mL} / \mathrm{min} / 1.73 \mathrm{~m}^{2}$ or more within 12 months

Poorly controlled hypertension despite at least four agents at therapeutic doses

Known or suspected rare or genetic cause of CKD

Suspected renal artery stenosis

Acute kidney injury is common, affecting $20 \%$ of all emergency admissions, and is an important risk factor for the development of CKD. People should be monitored for the development or progression of CKD for at least 2-3 years after an episode of acute kidney injury, even if serum creatinine has returned to baseline. Good communication between secondary care physicians and primary care is essential to ensure that this follow-up can take place in the community. People who have had acute kidney injury should be informed that they are at increased risk of CKD developing or progressing.

\section{Classification of CKD}

Decreased GFR and increased ACR are associated with increased risk of adverse outcomes (including CKD progression, end-stage kidney disease, acute kidney injury, cardiovascular disease and mortality) independently of each other and of traditional cardiovascular risk factors. This forms the basis of the classification system shown in Fig 1. ${ }^{4,5}$ This classification system can be used to inform management, determine the intensity of monitoring and tailor patient education.

\section{Pharmacotherapy}

Treatment strategies in CKD are aimed at reducing CVD risk, delaying CKD progression, addressing complications of CKD and, where possible, managing the underlying cause. The treatment of specific causes of kidney disease, such as glomerulonephritis, is outside the scope of this guideline. 
Fig 1. Classification of chronic kidney disease using glomerular filtration rate and albumin to creatinine ratio categories. Adapted with permission from Kidney Disease: Improving Global Outcomes (KDIGO) CKD Working Group. KDIGO 2012 clinical practice guideline for the evaluation and management of chronic kidney disease. Kidney Int Suppl 2013:3:1-150 and from National Institute for Health and Care Excellence. Chronic kidney disease in adults: assessment and management: Clinical guideline [CG182]. NICE, 2014. ${ }^{a}$ = consider using eGRFcystatinC for people with CKD G3aA1; ACR = albumin to creatinine ratio; $\mathrm{CKD}=$ chronic kidney disease:

eGFR = estimated glomerular filtration rate; $\mathrm{GFR}=$ glomerular filtration rate.

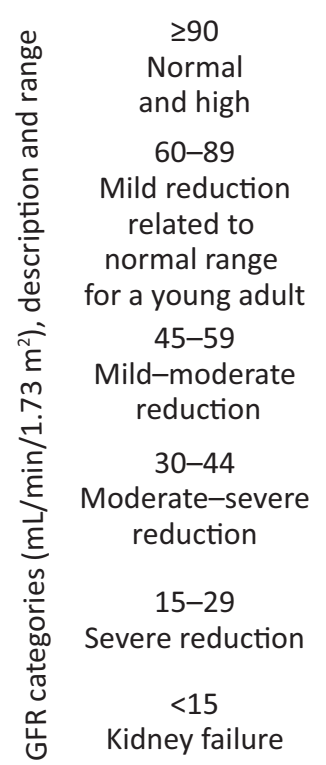

have been discontinued. A combination of renin-angiotensin system antagonists should not be offered to people with CKD. Hypertension in people with CKD but without diabetes or $A C R \geq 30 \mathrm{mg} / \mathrm{mmol}$ should be managed according to the treatment recommendations in NICE guideline Hypertension in adults: diagnosis and management: NICE guideline [NG136]. ${ }^{14}$

\section{Other strategies for renal protection}

There is some evidence that treatment of chronic metabolic acidosis with oral sodium bicarbonate may slow the progression to end-stage kidney disease. ${ }^{15}$ Consider oral sodium bicarbonate supplementation for people with both:

\section{Table 2. Blood pressure targets in chronic kidney}

\section{disease}

\begin{tabular}{ll} 
CKD & BP $120-139 /<90 \mathrm{mmHg}$ \\
CKD and diabetes & BP $120-129 /<80 \mathrm{mmHg}$ \\
CKD and ACR $\geq 70 \mathrm{mg} / \mathrm{mmol}$ & BP $120-129 /<80 \mathrm{mmHg}$ \\
\hline $\begin{array}{l}\text { ACR }=\text { albumin to creatinine ratio; BP }=\text { blood pressure; CKD }=\text { chronic kidney } \\
\text { disease. }\end{array}$
\end{tabular}

G1

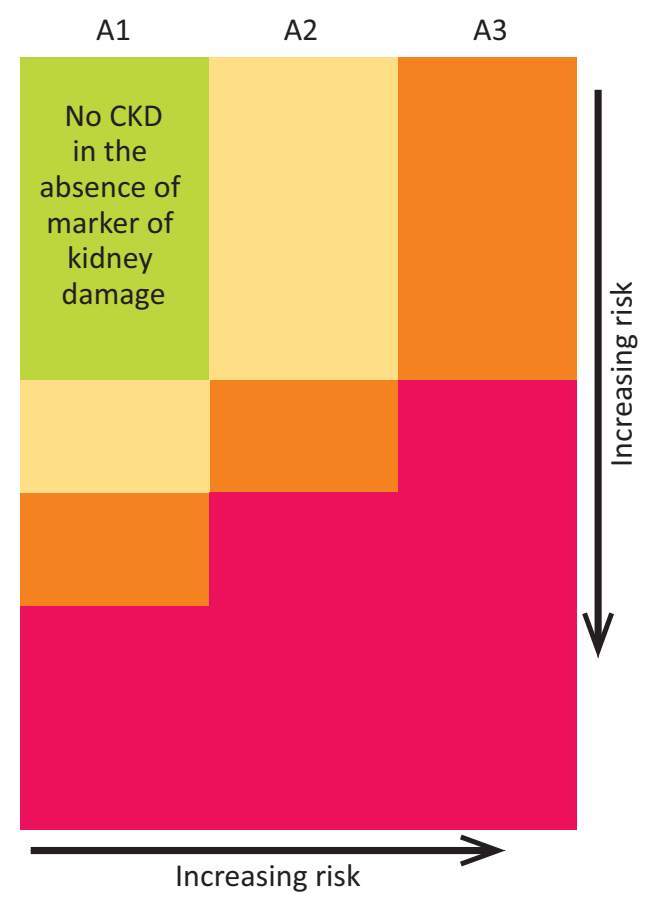

\begin{tabular}{ccc}
\multicolumn{3}{c}{ ACR categories $(\mathrm{mg} / \mathrm{mmol})}$, \\
descripton and range \\
$<3$ & $3-30$ & $>30$ \\
$\begin{array}{c}\text { Normal to } \\
\text { mildly }\end{array}$ & $\begin{array}{c}\text { Moderately } \\
\text { increased }\end{array}$ & $\begin{array}{c}\text { Severely } \\
\text { increased }\end{array}$ \\
increased &
\end{tabular}

A1

G1

G3a

G3b
> a GFR $<30 \mathrm{~mL} / \mathrm{min} / 1.73 \mathrm{~m}^{2}$ and

$>$ a serum bicarbonate concentration $<20 \mathrm{mmol} / \mathrm{L}$.

It is well established that glycaemic control in patients with diabetes mellitus can slow the development of albuminuria and CKD progression. ${ }^{16,17}$ There is also more recent evidence of a role for sodium-glucose co-transporter-2 inhibitors in reducing proteinuria and slowing the progressing of CKD in patients with type 2 diabetes. ${ }^{18} \mathrm{~A}$ detailed discussion of these findings is outside the scope of this article.

\section{Cardiovascular risk reduction}

Lipid lowering is important in CKD to reduce cardiovascular risk. Clinicians should follow the recommendations in NICE guideline Cardiovascular disease: risk assessment and reduction, including

Box 1. Indications for renin-angiotensin system antagonists in chronic kidney disease

Diabetes and $A C R \geq 3 \mathrm{mg} / \mathrm{mmol}$

Hypertension and $A C R \geq 30 \mathrm{mg} / \mathrm{mmol}$

$A C R \geq 70 \mathrm{mg} / \mathrm{mmol}$ irrespective of hypertension or CVD

$\mathrm{ACR}=$ albumin to creatinine ratio; $\mathrm{CVD}=$ cardiovascular disease. 
lipid modification: Clinical guideline [CG181], which recommends that, for primary and secondary prevention, atorvastatin should be offered to all people with CKD. ${ }^{19}$

Anti-platelet drugs should be offered to people with CKD for secondary prevention of cardiovascular disease, but clinicians should be aware of the increased risk of bleeding in this population.

Apixaban should be considered in preference to warfarin in people with eGFR $30-50 \mathrm{~mL} / \mathrm{min} / 1.73 \mathrm{~m}^{2}$ and non-valvular atrial fibrillation who have one or more of the following risk factors:

$>$ prior stroke or transient ischaemic attack

$>$ age $>75$ years old

$>$ hypertension

$>$ diabetes mellitus

> symptomatic heart failure.

\section{Bone metabolism and osteoporosis}

Serum calcium, phosphate, parathyroid hormone and vitamin D levels should not be routinely measured in people with a GFR $\geq 30 \mathrm{~mL} / \mathrm{min} / 1.73 \mathrm{~m}^{2}$; they should be measured in those with a GFR $<30 \mathrm{~mL} / \mathrm{min} / 1.73 \mathrm{~m}^{2}$.

Bisphosphonates should be offered if indicated for the prevention and treatment of osteoporosis in people with a GFR $\geq 30 \mathrm{~mL} / \mathrm{min} / 1.73 \mathrm{~m}^{2}$.

\section{Vitamin D supplements}

Vitamin D supplements should not be routinely offered to manage or prevent CKD-mineral and bone disorders. Colecalciferol or ergocalciferol should be offered to treat vitamin D deficiency in people with CKD and vitamin D deficiency. If vitamin D deficiency has been corrected, and symptoms of CKD-mineral and bone disorders persist, alfacalcidol or calcitriol should be offered to people with a GFR $<30 \mathrm{~mL} / \mathrm{min} / 1.73 \mathrm{~m}^{2}$. Serum calcium and phosphate concentrations should be regularly monitored in people receiving these drugs.

\section{Anaemia}

Haemoglobin level should be measured in people with GFR $<45$ $\mathrm{mL} / \mathrm{min} / 1.73 \mathrm{~m}^{2}$ to identify anaemia $(\mathrm{Hb}<110 \mathrm{~g} / \mathrm{L})$ and managed according to the NICE guideline Chronic kidney disease: managing anaemia: NICE guideline [NG8]. ${ }^{20}$

\section{Limitations}

Several areas for future research have been identified.

> Self-management: the impact of educational and supportive interventions to people with CKD on clinical outcomes.

> Anti-platelet therapy: the clinical and cost-effectiveness of low-dose aspirin compared with placebo for primary prevention of CVD in people with CKD. This is the subject of the current Aspirin to Target Arterial Events in CKD (ATTACK) trial. ${ }^{21}$

$>$ Renin-angiotensin system antagonists: the clinical and cost effectiveness of these agents in people aged $>75$ years with CKD.

$>$ Uric acid-lowering agents: the clinical and cost effectiveness of uric acid-lowering drugs on progression of CKD and mortality.
$>$ Vitamin D supplements in the management of CKD-mineral and bone disorders: the impact of treatment with vitamin $D$ or vitamin $\mathrm{D}$ analogues on patient-related outcomes.

\section{Implications for implementation}

The NICE guideline identifies three areas that may have a significant impact on practice or be challenging to implement.

\section{Calculating estimates of creatinine-based GFR}

Estimation of GFR using the CKD-EPI equation rather than the previously used MDRD Study equation has required laboratories to change their practice. Moving to CKD-EPI creatinine equation to estimate GFR may make it difficult to access trends over time in people with previous GFR estimates calculated using other equations.

\section{Using cystatin C-based estimates of GFR}

eGFRcystatinC is an additional diagnostic tool that may reduce over-diagnosis of CKD in people in primary care with a borderline diagnosis. It is not yet widely available and laboratories will need to invest in appropriate training and, in some cases, equipment (although it can be performed using existing analysers). Using cystatin $\mathrm{C}$ as an additional test will have financial implications (additional costs of testing but cost savings through reduced management costs). It is a relatively new recommendation and so clinicians may not be aware of when and how to request the test.

\section{Classifying CKD}

Assessing GFR and ACR may add additional burden and cost to diagnosis and monitoring of CKD. The addition of ACR increases the complexity of the classification and clinicians will need to be informed and educated regarding this and its clinical significance together with other developments as per planned guidance updates. ${ }^{22}$

\section{Conflicts of interest}

Hugh Gallagher was a member of the CG182 Guideline Development Group and is co-chief investigator of the ATTACK trial.

\section{Acknowledgments}

The contribution of all Guideline Development Group members to the development and review of Clinical Guideline 182 is gratefully acknowledged.

\section{References}

1 Roderick P, Roth M, Mindell J. Prevalence of chronic kidney disease in England: Findings from the 2009 Health Survey for England. J Epidemiol Community Health 2011;65:A12.

2 Muntner P, He J, Hamm L, Loria C, Whelton PK. Renal insufficiency and subsequent death resulting from cardiovascular disease in the United States. J Am Soc Nephrol 2002;13:745.

3 Kerr M, Bray B, Medcalf J, O'Donoghue DJ, Matthews B. Estimating the financial cost of chronic kidney disease to the NHS in England. Nephrology Dialysis Transplantation 2012;27:73-80. 
4 National Institute for Health and Care Excellence. Chronic kidney disease in adults: assessment and management: Clinical guideline [CG182]. NICE, 2014.

5 Kidney Disease: Improving Global Outcomes (KDIGO) CKD Working Group. KDIGO 2012 clinical practice guideline for the evaluation and management of chronic kidney disease. Kidney Int Suppl 2013:3:1-150.

6 Jacobson HR. Chronic renal failure: pathophysiology. Lancet 1991;338:419.

7 Kunz R, Friedrich C, Wolbers M, Mann JF. Meta-analysis: effect of monotherapy and combination therapy with inhibitors of the renin angiotensin system on proteinuria in renal disease. Ann Intern Med 2008; $148: 30$

8 Lv J, Ehteshami P, Sarnak MJ et al. Effects of intensive blood pressure lowering on the progression of chronic kidney disease: a systematic review and meta-analysis. CMAJ 2013;185:949-57.

9 Jafar TH, Stark PC, Schmid CH et al. Progression of chronic kidney disease: Blood pressure control, proteinuria, and angiotensinconverting enzyme inhibition. Ann Intern Med 2003:139:244-52.

10 Berl T, Hunsicker LG, Lewis JB et al. Impact of achieved blood pressure on cardiovascular outcomes in the Irbesartan Diabetic Nephropathy Trial. J Am Soc Nephrol 2005:16:2170.

11 Keane WF, Brenner BM, de Zeeuw D et al. The risk of developing end-stage renal disease in patients with type 2 diabetes and nephropathy: the RENAAL study. Kidney Int 2003;63:1499.

12 de Zeeuw D, Remuzzi G, Parving HH et al. Proteinuria, a target for renoprotection in patients with type 2 diabetic nephropathy: lessons from RENAAL. Kidney Int 2004;65:2309.

13 Jafar $\mathrm{TH}$, Schmid $\mathrm{CH}$, Landa M et al. Angiotensin-converting enzyme inhibitors and progression of nondiabetic renal disease. A meta-analysis of patient-level data. Ann Intern Med 2001;135:73.

14 National Institute for Health and Care Excellence. Hypertension in adults: diagnosis and management: NICE guideline [NG136]. NICE, 2019.
15 de Brito-Ashurst I, Varagunam M, Raftery M], Yaqoob MM. Bicarbonate supplementation slows progression of CKD and improves nutritional status. J Am Soc Nephrol 2009;20:2075.

16 DCCT/EDIC Research Group, de Boer IH, Sun W et al. Intensive diabetes therapy and glomerular filtration rate in type 1 diabetes. N Engl J Med 2011;365:2366.

17 UK Prospective Diabetes Study (UKPDS) Group. Intensive bloodglucose control with sulphonylureas or insulin compared with conventional treatment and risk of complications in patients with type 2 diabetes (UKPDS 33). Lancet 1998;352:837-53.

18 Neuen B, Young T, Heerspink HJL et al. SGLT2 inhibitors for the prevention of kidney failure in patients with type 2 diabetes: a systemic review and meta-analysis. Lancet 2019;7;845-54.

19 National Institute for Health and Care Excellence. Cardiovascular disease: risk assessment and reduction, including lipid modification: Clinical guideline [CG181]. NICE, 2014.

20 National Institute for Health and Care Excellence. Chronic kidney disease: managing anaemia: NICE Guideline [NG8]. NICE, 2015.

21 Aspirin to Target Arterial Events in Chronic Kidney Disease (ATTACK) trial. ClinicalTrials.gov, 2019. https://clinicaltrials.gov/ct2/ show/NCT03796156

22 National Institute for Health and Care Excellence. Chronic kidney disease: assessment and management (update): In development [GID-NG10118]. NICE. [in development]. www.nice.org.uk/guid ance/indevelopment/gid-ng10118 [Accessed 08 February 2020]

Address for correspondence: Prof Hugh Gallagher, St Helier Hospital, Wrythe Lane, Carshalton, Surrey SM5 1AA, UK. Email: hugh.gallagher1@nhs.net

\section{Royal College} of Physicians

\section{Guidance on safe medical staffing}

The RCP's Guidance on safe medical staffing working party report aims to help those planning and organising core hospital medical services to answer the question: 'How many doctors or their alternatives, with what capabilities, do we need to provide safe, timely and effective care for patients with medical problems?'.

\section{Download the report at: www.rcplondon.ac.uk/safe-medical-staffing}

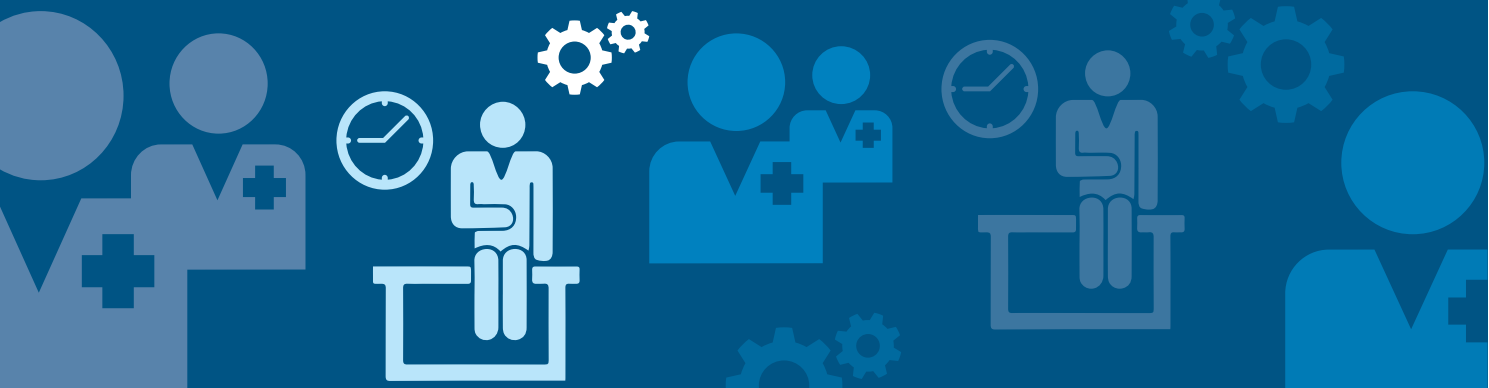

